Unternehmensbewertung unter veränderten Vorzeichen - Einführung in das Schwerpunktthema

\title{
Globalisierung: Unternehmen zwischen Macht- und Verantwortungszuwachs
}

D ie Zunahme der wel Verflechtungen prägte die letzten Jahrzehnte. Dies betrifft die Handels- und Kapital-, aber auch die Investitionsströme. Dieser Prozess, allgemein Globalisierung genannt, ist kein allein naturwüchsiger Prozess. Er war und ist politisch gewollt, weil Wohlfahrtsgewinne durch Deregulierung und Entpolitisierung und damit Vergrößerung der Märkte erhofft werden.

Im Rahmen dieser Globalisierungsprozesse haben sich die Gewichte der unterschiedlichen Akteure verändert. Dabei haben sich vor allem die Handlungsmöglichkeiten insbesondere der transnationalen Konzerne deutlich erhöht. Sie können jetzt nationalen Regulierungen ausweichen und damit die regulativen Regimes der Nationalstaaten miteinander in den Wettbewerb setzen, wie z.B. im Rahmen des Standort- oder Steuerwettbewerbs. Die Bewertung dieser Entwicklungen ist durchaus konträr: Wird auf der einen Seite dieser Wettbewerb als eine Möglichkeit der Bändigung des „Leviathans“ angesehen, so wird, durchaus auch von liberaler Seite (1), darauf verwiesen, dass die Staaten auch Anbieter öffentlicher Güter sind und eine Übertragung unternehmerischer Konzepte auf den Staat nur begrenzt Sinn macht.

Allerdings ist eine Verklärung nationalstaatlicher Politiken nicht angebracht. Die globalen Umweltprobleme sind trotz deutlich größerer nationalstaatlicher Regulierungsmöglichkeiten entstanden, die wirtschaftliche Globalisierung holt insofern ,nur" nach was im Umweltbereich bereits Realität ist.

Im Rahmen der weltwirtschaftlichen Integrationsprozesse steht die negative Integration im Vordergrund: es werden zum Beispiel im Rahmen der Welthandelsorganisation (WTO) vielfach nationale Regelwerke außer Kraft gesetzt. Eine Dynamik der positiven Integration, also des Setzens gemeinsamer Spielregeln, konnte im Rahmen von Umwelt- oder gar Sozialregimes auf der globalen Ebene bislang nur begrenzt erreicht werden. Hier sind in starkem Maße Ungleichgewichte im Verhältnis zur Dynamik der Liberalisierungsprozesse festzustellen.

Sowohl für die globalen Umweltprobleme als auch für die veränderten wirtschaftlichen Strukturen werden damit Regulierungsstrukturen auf einer höheren als allein der nationalstaatlichen Ebene erforderlich. Diese Regimes bilden sich nicht automatisch. Sie bedürfen vielmehr der politischen Steuerung aber auch der zivilgesellschaftlichen Einmischung, um eine Anpassung der existierenden Strukturen an die veränderten Verhältnisse zu erreichen.

\section{Fortentwicklung des Rahmens}

Vorschläge zur Fortentwicklung des ökologischen und sozialen Rahmens des Wirtschaftens existieren vielfältig und sind zumindest im Sinne von Anforderungen im Rahmen des Brandt Reports zur Global Governance identifiziert worden (2). Neueren Datums sind institutionelle Entwicklungsvorstellungen, wie sie etwa von Simonis entwickelt wurden (3). Es ist mehr oder weniger Einvernehmen über die Notwendigkeit eines solchen Rahmens festzustellen.

Aus eher statischer ordnungspolitischer Sicht ist zur Durchsetzung dieses Rahmens ein zentraler Akteur erforderlich. Dies erscheint in einer multipolaren Welt eine nicht adäquate - und durchaus auch nicht anstrebenswerte - Vorstellung zu sein. Vielmehr ist davon auszugehen, dass die erforderlichen Governance-Strukturen nicht allein von den Nationalstaaten geprägt sein werden. Die Zahl der Akteure nimmt zu und ihre Gewichte verschieben sich; neue Akteure müssen eingebunden werden. Gleichwohl erweist sich die Umsetzung entsprechender Strukturen als schwierig (4).

Die Entwicklung von Global-Governance-Strukturen muss insofern als langfristiger Prozess begriffen werden, bei dem nicht allein die Staaten, sondern auch zivilgesellschaftliche Akteure und Unternehmen eine wesentliche Rolle spielen müssen. Gerade die multinationalen Unternehmen haben mit einer wirtschaftlichen Macht, die eine Vielzahl von Nationalstaaten als wirt- schaftliche Zwerge erscheinen lässt, einen Stellenwert erreicht, der ihnen letztlich eine besondere Verpflichtung auferlegt. Diese implizite Verpflichtung, sich gegenüber der Zivilgesellschaft rechtfertigen zu können, hat auch eine reale Basis: die Furcht vor Imageverlusten und damit auch vor ökonomischen Verlusten.

\section{- Multis: Einschätzung im Wandel}

Die Bewertung der Aktivitäten der multinationalen Unternehmen hat sich in den letzten vierzig Jahren mehrfach gewandelt. In den 60er Jahren gerieten die Multis nicht zuletzt aufgrund der Ereignisse in Chile und des Einflusses auf die Entwicklungsländer stark in die Kritik. Dies führte in der Folge zu Verhandlungen auf UN-Ebene über einen verpflichtenden Code of Conduct on Transnational Corporations $(\mathrm{CoC})$, die aber letztich 1992 scheiterten. Die Einschätzung der Multis hat sich aber in den 80er und 90er Jahren deutlich gewandelt. Multinationale Unternehmen und insbesondere deren Direktinvestitionen wurden mehr und mehr als wesentliche Promotoren der wirtschaftlichen Entwicklung gesehen, der Konflikt schien dabei entschärft zu sein. Erst seit Ende der 90er Jahre scheint die Bewertung der multinationalen Unternehmen wiederum einem Wandel unterworfen zu sein. Nicht zuletzt die gescheiterten Verhandlungen um das multilaterale Investitionsabkommen sowie der WTO in Seattle markieren einen Stimmungswandel. Dieser wird von Anzeichen eines zunehmenden Anti-Corporatism begleitet (5). Die Rolle der multinationalen Unternehmen hat somit unterschiedliche Facetten. Auf der einen Seite verfügen sie über die Mittel, die Nationalstaaten herauszufordern und damit Wettbewersbsprozesse zwischen den Staaten auszulösen und zu einem race to the bottom beizutragen. Auf der anderen Seite verfügen sie zugleich über die Fähigkeit, auch jenseits der jeweiligen nationalstaatliche Regelungen Umwelt- und Sozialstandards durchzusetzen, was zu einem race to the top beitragen kann.

Mit der Forderung an die multinationalen Unternehmen, Umwelt- und Sozialstandards zu beachten, wird letztlich zunächst auf das oben beschriebene Defizit der Entwicklung des globalen Rahmens des Wirtschaftens verwiesen. Dementsprechend wird den multinationalen Unternehmen vielfach von Seiten wichtiger zivilgesellschaftlicher Akteure eine wesentliche Verantwortung zugewiesen, in ihren eigenen 
Produktionsstätten bzw. bei ihren Zulieferern entsprechende Anforderungen $\mathrm{zu}$ stellen um eben diese Standards durchzusetzen.

\section{Chancen für Unternehmen und die Zivilgesellschaft}

Die Unternehmen können sich als Akteure eines geschmeidigeren Wandels erweisen, indem sie gesellschaftliche Diskussionsprozesse bereits frühzeitig aufgreifen, die im politischen Entscheidungssystem nicht durchsetzbar sind. Ein wesentliches Instrument dazu können Selbstverpflichtungen sein, die allerdings einer Überprüfung zugänglich gemacht werden sollten. Hier kann eine sozial-ökologische Unternehmensbewertung ansetzen. Die Forderungen zivilgesellschaftlicher Akteure (Stakeholder) an die Unternehmen und deren Selbstverpflichtungen können die Entwicklung der internationalen Regimes unterstïtzen. Dies nicht zuletzt aufgrund des Eigeninteresses der Unternehmen selbst, die letztlich an der Entwicklung eines fairen Wettbewerbsrahmens interessiert sein müssen.

Vor diesem Hintergrund ist der Schwerpunkt auf Unternehmen als wesentliche Akteure des weltwirtschaftlichen Integrationsprozesses und die Möglichkeiten ihrer Beeinflussung ausgerichtet. Dabei werden vor allem Ansätze der sozialökologischen Unternehmensbewertung sowie die Möglichkeiten und Grenzen der Umweltund Sozialberichterstattung diskutiert.

\section{Die Beiträge im Überblick}

Zunächst beleuchtet der Beitrag von Wally Olins, einem der führenden internationalen Berater im Bereich Corporate Identity, vor dem Hinter- grund der Globalisierung und des Bedeutungszuwachses der multinationalen Unternehmen mögliche Auswirkungen auf die Rolle der Unternehmen. Diese stehen vor neuen Herausforderungen, die denen von Nationalstaaten, so seine These, zunehmend ähnlicher werden.

Um hierzu im Sinne von Global-GovernanceStrukturen ein zivilgesellschaftliches Gegengewicht $\mathrm{zu}$ setzen, sind Informationen und deren Verarbeitung eine Grundvoraussetzung. Mit der möglichen Überforderung der Stakeholder der Unternehmen in ihrer neuen Rolle setzt sich Jürgen Freimann auseinander. Trotzdem sieht er die Erfordernis einer umfassenden Transparenz der Unternehmen und entsprechender Bewertungsverfahren. Die Kapitalmärkte können hierfür sogar eine Katalysatorrolle spielen.

Die Vielfalt der Formen externer sozial-ökologische Unternehmensbewertungen als Steuerungsund Interventionsinstrumente der Zivilgesellschaft, von Verbraucherboykotten bis zu wissenschaftlich fundierten vergleichenden Bewertungen, stellt Volkmar Lübke dar.

Wie sieht aber die Verbreitung von Codes of Conduct in der Unternehmenspraxis aus? Am Beispiel Umwelt nehmen die OECD-AutorInnen Maiko Miyake und Kathryn Gordon eine internationale Auswertung der entsprechenden CoCs und der Berichterstattung darüber vor. Der Beitrag zeigt die Entwicklungsdynamik auf und setzt sie zu internationalen Regimes in Bezug.

Das Lenkungssystem Öffentlichkeit und die damit zusammenhängenden Wirkungsmechanismen und Lernschleifen innerhalb und außerhalb der Unternehmen stehen im Mittelpunkt des abschließenden Beitrages von Jens Clausen . Er sieht die Nachhaltigkeitsberichterstattung als eine notwendige Ergänzung sozial-ökologischer CoC's, da nur durch eine öffentliche Rechenschaftslegung die gesellschaftliche Diskussion über die Unternehmen in Gang gesetzt und in Bewegung gehalten werden kann. Ergänzt wird der Schwerpunkt durch einen Überblick über die wichtigsten derzeit aktiven sozial-ökologischen Bewertungsinstitutionen.

\section{Anmerkungen}

(1) Sinn, H.W.: The selection principle and market failure ins systems competition. In: Journal of Public Economics Vol. 66 (1997), S. 247-274.

(2) Stiftung Entwicklung und Frieden (Hrsg.): Nachbarn in Einer Welt. Der Bericht der Kommission für Weltordnungspolitik. Bonn 1995. Vergleiche hierzu auch den Schwerpunkt Global Governance und Umweltpolitik in Ökologisches Wirtschaften Nr. 2/1998.

(3) Vgl. z.B. Biermann, F./ Simonis, U.E.: Eine Weltorganisation für Umwelt und Entwicklung. Policy Paper Nr. 9. SEF. Bonn 1998.

(4) Dies wurde in der Zwischenbilanz zum Brandt Report deutlich. Vgl. Nuscheler, F. et al.: Entwicklung und Frieden im 21 Jh.. Dietz Verlag, Bonn 2000.

(5) Vgl. z.B. Korten, D.C.: When Corporations rule the World. London 1995. In neuerer Zeit z.B. Klein, N.: No Logo. Picador 1999.

\section{Die Autoren}

Ulrich Petschow und Jens Clausen sind wissenschaftliche Mitarbeiter am Institut für ökologische Wirtschaftsforschung.

Kontakt: IÖW, Potsdamer Str. 105, 10785 Berlin. Tel. 030/884594-0, Fax 030/ 8825439,

E-mail: Ulrich.Petschow@ioew.de, Jens.Clausen@ hannover.ioew.de

\title{
punkt-um.net
}

\section{Ihre Internet-Plattform zum Infodienst punkt.um für Informationen zu Nachhaltigkeit und Ökologie}

\author{
Aktuelle Termine - News aus dem Internet - Website-Datenbank \\ Ökomedia: Abstracts-Datenbank zu 70.000 Zeitschriftenartikeln und Bücher seit 1987
}

Sie finden uns unter: http://www.punkt-um.net 
(c) 20I0 Authors; licensee IÖW and oekom verlag. This is an article distributed under the terms of the Creative Commons Attribution Non-Commercial No Derivates License (http://creativecommons.org/licenses/by-nc-nd/3.o/), which permits unrestricted use, distribution, and reproduction in any medium, provided the original work is properly cited. 